\title{
GENERALIZED $\beta$-TRANSFORMATIONS AND THE ENTROPY OF UNIMODAL MAPS
}

\author{
DANIEL J. THOMPSON
}

\begin{abstract}
Generalized $\beta$-transformations are the class of piecewise continuous interval maps given by taking the $\beta$-transformation $x \mapsto \beta x$ $(\bmod 1)$, where $\beta>1$, and replacing some of the branches with branches of constant negative slope. If the orbit of 1 is finite, then the map is Markov, and we call $\beta$ (which must be an algebraic number) a generalized Parry number. We show that the Galois conjugates of such $\beta$ have modulus less than 2 , and the modulus is bounded away from 2 apart from the exceptional case of conjugates lying on the real line. We give a characterization of the closure of all these Galois conjugates, and show that this set is path connected. Our approach is based on an analysis of Solomyak for the case of $\beta$-transformations. One motivation for this work is that the entropy of a post-critically finite (PCF) unimodal map is the logarithm of a generalized Parry number. Thus, our results give a mild restriction on the set of entropies that can be attained by PCF unimodal maps.
\end{abstract}

\section{INTRODUCTION}

For a continuous post-critically finite (PCF) interval map, the exponential of the topological entropy, denoted $\exp h$, is a Perron number. Thurston showed that all Perron numbers can be obtained this way [23]. However, for PCF multimodal maps with restricted degree, the situation changes dramatically: the dynamics impose complicated restrictions on which Perron numbers can be attained as $\exp h$. We want to describe these numbers by understanding the restrictions on the Galois conjugates of $\exp h$.

This problem was raised in Thurston's final paper [23, which includes a figure of the set of complex numbers which are Galois conjugates of exp $h$ for PCF unimodal maps. We denote this set by $\Omega_{T}$. An ongoing problem raised by Thurston's final paper is to understand the structure of $\Omega_{T}$. So far, progress has been made by Calegari, Koch and Walker on understanding the region of $\Omega_{T}$ which lies inside the unit disk [5], and Tiozzo [24] has shown that the set $\overline{\Omega_{T}}$ is path connected. There has been no further progress on understanding the region of $\Omega_{T}$ which lies outside the unit disk.

Date: January 12, 2017.

2000 Mathematics Subject Classification. 37E05, 37B40, 11R06, 30C15.

This work is supported by NSF grant DMS-1461163. 
In this paper, we study an analogous problem for a class of interval maps called generalized $\beta$-transformations, and use this to gain at least some information about the outer boundary of $\Omega_{T}$.

Our approach is based on the formalism of generalized $\beta$-transformations, as introduced by Góra [10]. The generalized $\beta$-transformations are the class of piecewise continuous interval maps given by replacing some of the branches of a $\beta$-transformation with branches of constant slope $-\beta$. We call a generalized $\beta$-transformation post-critically finite (PCF) if the point 1 has a finite orbit, to unify terminology with the case of continuous multimodal maps. We let $\Omega$ denote the set of Galois conjugates of all $\beta$ such that there exists a PCF generalized $\beta$-transformation.

The class of generalized $\beta$-transformations contains all $\beta$-transformations, and many continuous interval maps. Of particular interest is the case where $\beta \in(1,2)$, the first branch is increasing, and the second branch is decreasing. This gives a class of continuous unimodal maps among which the entropy of every PCF unimodal map is represented (i.e. every PCF unimodal map is semi-conjugate to a PCF map in this class with the same entropy). In particular, we can conclude that $\Omega_{T} \subset \Omega$, see $₫ 6$ for more details.

We show that $\Omega$ lies inside a disk of radius 2. Since $\exp h$ and the degree of a (continuous) generalized $\beta$-transformation can be arbitrarily large, this result contrasts sharply with the result that any Perron number can be achieved as the entropy of a general PCF multimodal map.

Although it is trivial that $\Omega_{T}$ lies inside a disk of radius 2 , since unimodal maps satisfy $\exp h \leq 2$, our results can be improved by excluding the exceptional case of real-valued Galois conjugates. Experimental investigation of Beaucoup, Borwein, Boyd and Pinner [2] suggests that a sharp bound should be less than 1.6. We show rigorously that all non-real-valued Galois conjugates have modulus bounded away from 2 , at least establishing the principle that $\Omega_{T} \backslash \mathbb{R}$ lies in a disk of radius less than 2 .

Our techniques are inspired by an analysis of Solomyak [20]. For 'PCF' $\beta$-transformations, i.e. those for which the point 1 has a finite orbit, in which case we call $\beta$ a Parry number, Solomyak showed that all Galois conjugates are bounded in modulus by the golden mean, and that this bound is sharp, improving on a bound of 2 obtained by Parry [16]. Furthermore, Solomyak established a Structure Theorem which gives a rather explicit characterization of the largest Galois conjugate in each direction (i.e. with a prescribed argument). Our approach is based on extending these results as far as possible to the setting of PCF generalized $\beta$-transformations. In particular, we have a version of the Solomyak Structure Theorem for generalized $\beta$-transformations. This result provides an analytic tool for studying the largest modulus of points in $\Omega$, and thus for bounding above the largest modulus of points in $\Omega_{T}$. Showing that this theory applies in the context of $\Omega_{T}$ is one of the main points of this article, as no techniques were previously available for attacking this problem. 
The main idea of the argument is to characterize those elements of $\Omega$ with $|z|>1$ as the inverse of a zero of an analytic function in the class $\mathcal{F}=\left\{T(w)=1+\sum_{j=1}^{\infty} a_{j} w^{j}: a_{j} \in[-1,1]\right\}$. This correspondence is obtained from the expression for the generalized $\beta$-expansion of 1 , and makes the problem tractable to further analysis. We also show that if $\lambda$ is a zero of a function in $\mathcal{F}$, then $\lambda^{-1} \in \bar{\Omega}$. We use this to show that $\bar{\Omega}$ is path connected.

In $\S 2$, we introduce generalized $\beta$-transformations. In $\oint 3$, we introduce generalized Parry numbers and generalized Parry polynomials, and obtain our basic bound on the size of $\Omega$. In $\$$, we establish a description of the outer boundary of $\Omega$ and establish slightly improved bounds on $\Omega \backslash \mathbb{R}$. In 95. we study $\bar{\Omega}$. In $\$ 6$, we apply our results to unimodal maps.

\section{Generalized $\beta$-transformations}

The $\beta$-transformations are the class of piecewise continuous interval maps $x \rightarrow \beta x(\bmod 1)$, where $\beta>1$. The class of generalized $\beta$-transformations, introduced by Góra [10], are obtained from the $\beta$-transformations by flipping some of the branches so the slope is $-\beta$, and extending the map to a piecewise continuous map of the closed interval $[0,1]$. It is clear what it means to flip a full branch of the map. If we flip the rightmost branch, which is the only branch that is not full, we mean that this 'flipped branch' of the map decreases from 1 to $1-\{\beta\}$, where $\{\beta\}$ is the fractional part of $\beta$. The precise definition is given below. We record the configuration of positive and negative slopes by a vector $E$ of 1 's and -1's. The 1's correspond to increasing branches, and the -1 's correspond to decreasing branches. There is a large literature on using classes of interval maps to give expansions of real numbers [17, 6, 7, 22, 18. Generalized $\beta$-transformations were introduced in this context. Connections with the theory of tilings are given in [9].

The $(1,-1)$ case. A case of particular interest in this study is the sign configuration $E=(1,-1)$, in which case the map is a continuous unimodal map. For ease of exposition, we define the map rigorously in this case first. We let $\beta \in(1,2]$, and we let $I_{0}=[0,1 / \beta]$ and $I_{1}=(1 / \beta, 1]$, so that the sets $I_{0}, I_{1}$ denote the partition of $[0,1]$ into the two intervals of monotonicity. In this case, the generalized $\beta$-transformation has the formula

$$
f(x)= \begin{cases}\beta x & \text { if } x \in I_{0} \\ 2-\beta x & \text { if } x \in I_{1}\end{cases}
$$

For $j \geq 1$, we let

$$
d(x, j)= \begin{cases}0 & \text { if } f^{j-1} x \in I_{0} \\ 2 & \text { if } f^{j-1} x \in I_{1}\end{cases}
$$


The symbols $d(x, j)$ are the digits used in the generalized $\beta$-expansion of $x$. For $j \geq 1$, we let

$$
e(x, j)= \begin{cases}1 & \text { if } f^{j-1} x \in I_{0} \\ -1 & \text { if } f^{j-1} x \in I_{1}\end{cases}
$$

We define the 'cumulative sign' by $s(x, 1)=1$,

$$
s(x, j+1)=e(x, j) s(x, j)=\prod_{l=1}^{j} e(x, l),
$$

and we let $s(j):=s(1, j)$. The generalized $\beta$-expansion of $x$ is the expression

$$
x=\frac{s(x, 1) d(x, 1)}{\beta}+\frac{s(x, 2) d(x, 2)}{\beta^{2}}+\cdots+\frac{s(x, j) d(x, j)}{\beta^{j}}+\cdots
$$

By [10, Corollary 2], this expression is valid for every $x \in[0,1]$.

All generalized $\beta$-transformations. Let $m \in \mathbb{N}$ and $\beta \in(m, m+1]$. For such $\beta$, a generalized $\beta$-transformation has $m+1$ branches. We let $E=(E(0), E(1), \ldots, E(m)) \in\{1,-1\}^{m+1}$ be the vector which describes the configuration of slopes of the map (where an entry 1 corresponds to positive slope, and an entry -1 corresponds to negative slope). We partition $I$ into $m+1$ intervals

$$
I_{0}=\left[0, \frac{1}{\beta}\right], I_{1}=\left(\frac{1}{\beta}, \frac{2}{\beta}\right], \ldots, I_{m}=\left(\frac{m}{\beta}, 1\right],
$$

and we define the $(\beta, E)$-transformation $f=f_{\beta, E}$ by the formula

$$
f(x)= \begin{cases}\beta x-k & \text { if } x \in I_{k} \text { and } E(k)=1 \\ -\beta x+k+1 & \text { if } x \in I_{k} \text { and } E(k)=-1\end{cases}
$$

Note that the intervals $I_{j}$ are defined to include their right end-points, and $f$ is defined on the whole interval $[0,1]$. In the case that all entries of $E$ are 1 , then $f$ is an extension of the classical $\beta$-transformation $x \rightarrow \beta x(\bmod 1)$ to a piecewise continuous map of the closed interval $[0,1]$.

For $j \geq 1$, we let

$$
d(x, j)= \begin{cases}k & \text { if } f^{j-1} x \in I_{k} \text { and } E(k)=1 \\ k+1 & \text { if } f^{j-1} x \in I_{k} \text { and } E(k)=-1 .\end{cases}
$$

For $j \geq 1$, we let $e(x, j)=E(k)$ if $f^{j-1} x \in I_{k}$. We define the 'cumulative sign' by $s(x, 1)=1$,

$$
s(x, j+1)=e(x, j) s(x, j)=\prod_{l=1}^{j} e(x, l) .
$$

Again, Góra shows that for every $x \in[0,1]$,

$$
x=\frac{s(x, 1) d(x, 1)}{\beta}+\frac{s(x, 2) d(x, 2)}{\beta^{2}}+\cdots+\frac{s(x, j) d(x, j)}{\beta^{j}}+\cdots
$$


We refer to this expression as the $(\beta, E)$-expansion for $x$. For the $(\beta, E)$ expansion of 1 , we write $d(j):=d(1, j)$ and $s(j):=s(1, j)$.

We sometimes write $(\beta, E)$-expansions using sequence notation

$$
((s(x, 1), d(x, 1)),(s(x, 2), d(x, 2)),(s(x, 3), d(x, 3)), \ldots) .
$$

The set-up above includes the classic $\beta$-expansion simply by setting all entries in $E$ to be 1 . In this case, $s(x, j)=1$ for all $x$ and $j$, and (2.1) reduces to the standard $\beta$-expansion of Rényi and Parry.

2.1. Finite versus infinite $(\beta, E)$-expansions. It is possible in the definition of the $(\beta, E)$-expansion that there exists $n$ so that $d(x, j)=0$ for all $j>n$, and thus the $(\beta, E)$-expansion of $x$ is finite. This can only happen if $E(0)=1$, so 0 is a fixed point, and $f^{n} x=0$. Since the set of preimages of 0 is a subset of the left end-points of the intervals $I_{j}$, we must have $f^{n-1} x \in\{1 / \beta, 2 / \beta, \ldots,[\beta] / \beta\}$.

We explain how to derive an infinite $(\beta, E)$-expansion from a finite $(\beta, E)$ expansion. We start with the case that $x=1$ has a finite $(\beta, E)$-expansion. The finite $(\beta, E)$-expansion of 1 is thus

$$
1=\frac{s(1) d(1)}{\beta}+\frac{s(2) d(2)}{\beta^{2}}+\ldots+\frac{s(n) d(n)}{\beta^{n}},
$$

where $d(n) \neq 0$. Let $d^{\prime}(j)=d(j)$ for $j \in\{1, \ldots, n-1\}$, and

$$
d^{\prime}(n)= \begin{cases}d(n)-1 & \text { if } s(n)=1 \\ d(n)+1 & \text { if } s(n)=-1,\end{cases}
$$

noting that $0 \leq d^{\prime}(n) \leq d(1)$. This is because $1 \leq d(n)$, and $d(n) \leq d(1)$. It is easily checked that the only way we can have $d(n)=d(1)$ is if $\beta \in \mathbb{N}$, and $f(1)=0$. In this case, since $s(1)=1, d^{\prime}(n)=d^{\prime}(1)=d(1)-1$.

We have

$$
1=\frac{s(1) d^{\prime}(1)}{\beta}+\frac{s(2) d^{\prime}(2)}{\beta^{2}}+\ldots+\frac{s(n) d^{\prime}(n)}{\beta^{n}}+\frac{1}{\beta^{n}},
$$

and thus for any $m \geq 0$,

$$
\frac{1}{\beta^{m n}}=\sum_{j=1}^{n} \frac{s(j) d^{\prime}(j)}{\beta^{j+m n}}+\frac{1}{\beta^{(m+1) n}} .
$$

Note that by (2.3) and (2.4), we have

$$
1=\frac{s(1) d^{\prime}(1)}{\beta}+\ldots+\frac{s(n) d^{\prime}(n)}{\beta^{n}}+\frac{s(1) d^{\prime}(1)}{\beta^{n+1}}+\ldots+\frac{s(n) d^{\prime}(n)}{\beta^{2 n}}+\frac{1}{\beta^{2 n}} .
$$

Continuing this way, using (2.4), we obtain that for any $m \geq 1$,

$$
1=\sum_{j=0}^{m-1} \sum_{k=1}^{n} \frac{s(k) d^{\prime}(k)}{\beta^{k+j n}}+\frac{1}{\beta^{m n}},
$$


and it follows that $1=\sum_{j=1}^{\infty} s(j) d^{\prime}(j) / \beta^{j}$. This expression is the infinite $(\beta, E)$-expansion of 1 . Note that if 1 has a finite $(\beta, E)$-expansion, then the corresponding infinite $(\beta, E)$-expansion is periodic.

Now suppose that $x$ has a finite $(\beta, E)$-expansion. Then

$$
\begin{aligned}
x & =\frac{s(x, 1) d(x, 1)}{\beta}+\frac{s(x, 2) d(x, 2)}{\beta^{2}}+\ldots+\frac{s(x, k) d(x, k)}{\beta^{k}} \\
& =\frac{s(x, 1) d(x, 1)}{\beta}+\frac{s(x, 2) d(x, 2)}{\beta^{2}}+\ldots+\frac{s(x, k) d^{\prime}(x, k)}{\beta^{k}}+\frac{1}{\beta^{k}},
\end{aligned}
$$

where we define $d^{\prime}(x, k)=d(x, k)-1$ if $s(x, k)=1$, and $d^{\prime}(x, k)=d(x, k)+1$ if $s(x, k)=-1$. Thus, the infinite $(\beta, E)$-expansion of $x$ is given by the sequence $v w$, where

$$
v=\left((s(x, 1), d(x, 1)), \ldots,(s(x, k-1), d(x, k-1)),\left(s(x, k), d^{\prime}(x, k)\right)\right),
$$

and $w$ is the (infinite) $(\beta, E)$-expansion of 1 .

2.2. Space of Itineraries. There is another way to use $f$ to assign a sequence to a point: it is sometimes convenient to consider the itinerary of a point relative to the partition $\left\{I_{0}, \ldots, I_{m}\right\}$, where $\beta \in(m, m+1]$.

Let $\Sigma_{m}=\prod_{i=1}^{\infty}\{0, \ldots, m\}$. Given $x \in I$, its itinerary

$$
\operatorname{It}(x)=(\operatorname{It}(x, 1), \operatorname{It}(x, 2), \ldots)
$$

under $f=f_{\beta, E}$ is the sequence in $\Sigma_{m}$ given by

$$
\operatorname{It}(x, j)=i \text { if } f^{j-1} x \in I_{i} .
$$

Using the rules on the digits $d(x, i)$ and the signs $s(x, i)$, the $(\beta, E)$-expansion for $x$ can be recovered from $\operatorname{It}(x)$ and vice versa. In particular, we can map the $(\beta, E)$-expansion of 1 to the itinerary of 1 by the formula

$$
\operatorname{It}(1, j)= \begin{cases}d(j) & \text { if } s(j+1)=s(j) \\ d(j)-1 & \text { if } s(j+1)=-s(j) .\end{cases}
$$

We recall the criteria of Góra for determining the validity of itineraries, and hence $(\beta, E)$-expansions. First we define an order $<_{E}$ on $\Sigma_{m}$. Given a finite word $w(1) \cdots w(j)$ from the alphabet $\{0, \ldots, m\}$, we let $\operatorname{sign}_{E}(w):=$ $E(w(1)) \cdots E(w(j))$.

We define the ordering $\leq_{E}$ by declaring $w<_{E} v$ if $w(1)<v(1)$, or if $j$ is the first place where $w(j) \neq v(j)$, then

$$
w<_{E} v \text { if }\left\{\begin{array}{l}
w(j)<v(j) \text { if } \operatorname{sign}_{E}(w(1) \cdots w(j-1))=1 \\
w(j)>v(j) \text { if } \operatorname{sign}_{E}(w(1) \cdots w(j-1))=-1 .
\end{array}\right.
$$

The order $\leq_{E}$ also makes sense on the set of finite sequences $\prod_{i=1}^{k}\{0, \ldots, m\}$ for any fixed $k \geq 1$. Proposition 5 of Góra [10] says that a sequence $w \in$ $\Sigma_{m}$ is the itinerary of a point $x$ under $f_{\beta, E}$ if and only if for all $j \geq 0$, $\sigma^{j} w \leq_{E} \operatorname{It}(1)$. We remark that by taking the closure of the space of all such 
itineraries in $\Sigma_{m}$, this criteria can be thought of as determining the symbolic dynamics associated to $f_{\beta, E}$.

The order $\leq_{E}$ is an essential ingredient in the theory of one-dimensional maps, and has its roots in the work of Parry [17. This is a special case of the characterization of symbolic dynamics of piecewise monotonic maps that is formulated more generally in e.g. [8]. Similar ideas appear in the celebrated work of Milnor and Thurston [14] for continuous multimodal maps, where they assign to each point a sequence $\theta(x)$, called the invariant coordinate of $x$. For points $x$ that are not pre-images of a critical point, the sequence $\theta(x)$ is exactly determined by the itinerary and sign data $(s(x, i))_{i \in \mathbb{N}}$ of $x$.

2.3. Key identities for generalized $\beta$-transformations. First, we establish the fundamental relationship between the coefficients $d(j), s(j)$, and the 'signed orbit' of 1 which we write $c_{j}:=s(j+1) f^{j}(1)$. Note that $c_{0}=1$.

Lemma 2.1. For $j \geq 0$, the coefficients satisfy the recursion relation

$$
\beta c_{j}-s(j+1) d(j+1)=c_{j+1} \text {. }
$$

Proof. First, we rewrite the map $f$ as

$$
f(x)=e(x, 1)(\beta x-d(x, 1)),
$$

and thus

$$
f^{j}(x)=e(x, j)\left(\beta f^{j-1}(x)-d(x, j)\right) .
$$

In particular, we have $f^{j}(1)=e(1, j)\left(\beta f^{j-1}(1)-d(j)\right)$ for $j \geq 1$. Thus, for $j \geq 0$, we have

$$
\beta f^{j}(1)-d(j+1)=e(1, j+1) f^{j+1}(1) .
$$

Multiplying by $s(j+1)$ yields

$\beta s(j+1) f^{j}(1)-s(j+1) d(j+1)=s(j+1) e(1, j+1) f^{j+1}(1)=s(j+2) f^{j+1}(1)$, which establishes (2.5).

Now we prove an identity which is key to our analysis, generalizing an identity which was observed in Solomyak [20] for $\beta$-transformations.

Lemma 2.2. For any $z$ with $|z|>1$,

$$
1-\sum_{j=1}^{\infty} s(j) d(j) z^{-j}=\left(1-\frac{\beta}{z}\right) \sum_{j=0}^{\infty} c_{j} z^{-j}
$$

where $c_{j}=s(j+1) f^{j}(1)$.

Proof. We assume that $|z|>1$ so that the above series converge. Multiplying out the right hand side, we obtain

$$
\left(1-\frac{\beta}{z}\right) \sum_{i=0}^{\infty} c_{i} z^{-i}=1+\left(c_{1}-\beta\right) z^{-1}+\ldots+\left(c_{j+1}-c_{j} \beta\right) z^{-(j+1)}+\ldots
$$

For all $j \geq 0$, we have $c_{j+1}-c_{j} \beta=-s(j+1) d(j+1)$ by (2.5), which yields the required inequality. 
2.4. Post-critically finite generalized $\beta$-transformations. We define a generalized $\beta$-transformation to be post-critically finite $(P C F)$ if the orbit of 1 is finite, i.e. $\left\{f^{j}(1) \mid j \geq 0\right\}$ takes finitely many values. More generally, we say a piecewise monotonic map (not necessarily continuous) is post-critically finite if all maxima, minima and discontinuity points have a finite orbit. For a generalized $\beta$-transformation, all discontinuity points are pre-images of the points 1 or 0 , and 0 is either a fixed point or satisfies $f(0)=1$, so these definitions agree. We choose this terminology in order to be consistent with the literature on continuous multimodal maps, where PCF is the standard term for a map whose topological critical points have a finite orbit.

By the general theory of piecewise monotonic maps [14, 1], if the map is $\mathrm{PCF}$, then it admits a Markov partition. That is, there is a partition $\mathcal{P}$ of the interval into subintervals such that for all $P \in \mathcal{P}, \overline{f(P)}$ is the closure of a union of elements of $\mathcal{P}$. The partition $\mathcal{P}$ is obtained by taking subintervals whose endpoints are the forward orbits of the critical points. Thus, PCF interval maps are the ones that can be modeled by a shift of finite type, and thus have a well understood orbit structure. This motivates why we investigate which interval maps are PCF.

\section{Generalized Parry numbers and Parry polynomials}

We review the definition of a Parry number, and a Parry polynomial from the $\beta$-transformation literature, and extend these concepts to generalized $\beta$-transformations. Parry numbers and the Parry polynomial were both introduced in his seminal paper on $\beta$-expansions [16].

3.1. Parry numbers and Parry polynomials. A number $\beta>1$ is a Parry number if the $\beta$-expansion of 1 is pre-periodic. This occurs if and only if 1 has a finite orbit under $f_{\beta}$. We say that a Parry number is a simple Parry number if the $\beta$-expansion of 1 is periodic.

For a Parry number, the Parry polynomial $P_{\beta}(z)$ is a monic polynomial with integer coefficients which is naturally associated to the infinite $\beta$-expansion of 1 . We obtain $P_{\beta}$ by taking the infinite $\beta$-expansion of 1

$$
1=\sum_{j=1}^{\infty} \frac{d(j)}{\beta^{j}},
$$

and using the geometric series formula on the right hand side. We multiply through so all $\beta$ have a non-negative exponent, and bring all terms to one side. The resulting expression is the formula $P_{\beta}(\beta)=0$. For a simple Parry number with infinite $\beta$-expansion of 1 given by $(d(1), \ldots, d(p))^{\infty}$, we arrive at the expression

$$
\begin{aligned}
P_{\beta}(z) & =z^{p}-\sum_{j=1}^{p} d(j) z^{p-j}-1 \\
& =z^{p}-d(1) z^{p-1}-d(2) z^{p-2}-\cdots-d(p-1) z-1-d(p) .
\end{aligned}
$$


Since $P_{\beta}(\beta)=0$, all Galois conjugates of $\beta$ must also satisfy $P_{\beta}(z)=0$. The polynomial $P_{\beta}$ is not necessarily irreducible (i.e. it might have higher degree than the minimal polynomial for $\beta$ ), so $P_{\beta}$ may have zeros which are not Galois conjugates of $\beta$. Such a zero is called a $\beta$-conjugate. The distribution of $\beta$-conjugates was studied in [26, 25].

3.2. Generalized Parry numbers and Parry polynomials. We define a number $\beta>1$ to be a generalized Parry number if we can find $E$ so that the orbit of 1 under the $(\beta, E)$-transformation is finite. Thus, in the terminology of $\$ 2.4, \beta$ is a generalized Parry number iff there exists $E$ so that the $(\beta, E)$-transformation is $\mathrm{PCF}$.

Let $f=f_{\beta, E}$ be a PCF generalized $\beta$-transformation. Since the orbit of 1 is periodic or pre-periodic, then so is the sequence given by the infinite $(\beta, E)$-expansion of 1 .

Definition 3.1. For a post-critically finite $(\beta, E)$-transformation, let us write the infinite $(\beta, E)$-expansion of 1 as $v w^{\infty}$, where

$$
\begin{gathered}
v=((s(1), d(1)),(s(2), d(2)), \ldots,(s(k), d(k))), \\
w=((s(k+1), d(k+1)), \ldots,(s(k+p), d(k+p))) .
\end{gathered}
$$

In the above, $w$ is written with the lowest possible period, and in the periodic case $k=0, v$ is the empty word. We define the generalized Parry polynomial to be

$$
P_{\beta, E}(z)=z^{k+p}-\sum_{j=1}^{k+p} s(j) d(j) z^{k+p-j}-z^{k}+\sum_{j=1}^{k} s(j) d(j) z^{k-j} .
$$

The formula simplifies if the $(\beta, E)$-expansion of 1 is periodic, in which case

$$
P_{\beta, E}(z)=z^{p}-\sum_{j=1}^{p} s(j) d(j) z^{p-j}-1 .
$$

The expression $P_{\beta, E}(\beta)=0$ can be derived from applying the geometric series formula to the $(\beta, E)$-expansion of 1 , which motivates the definition of $P_{\beta, E}$. The following lemma is based on this relationship.

Lemma 3.1. A number $z$ with $|z|>1$ is a zero of $P_{\beta, E}(z)$ if and only if

$$
1-\sum_{j=1}^{\infty} s(j) d(j) z^{-j}=0 .
$$

Proof. We first assume that the infinite $(\beta, E)$-expansion of 1 is periodic. Suppose $z$ with $|z|>1$ satisfies $P_{\beta, E}(z)=0$. Then

$$
1-z^{-p}=\sum_{j=1}^{p} s(j) d(j) z^{-j}
$$


and thus

$$
1=\frac{\alpha}{1-z^{-p}},
$$

where $\alpha=\sum_{j=1}^{p} s(j) d(j) z^{-j}$. Using the geometric series formula yields

$$
1=\sum_{m=0}^{\infty} \alpha z^{-p m}=\sum_{m=0}^{\infty} \sum_{j=1}^{p} s(j) d(j) z^{-p m-j}=\sum_{j=1}^{\infty} s(j) d(j) z^{-j} .
$$

The general case follows the same strategy. Suppose now that the infinite $(\beta, E)$-expansion of 1 is pre-periodic, and $z$ with $|z|>1$ satisfies $P_{\beta, E}(z)=0$. Then

$$
z^{k+p}-\sum_{j=1}^{k} s(j) d(j) z^{k+p-j}-z^{k}+\sum_{j=1}^{k} s(j) d(j) z^{k-j}=\sum_{j=k+1}^{k+p} s(j) d(j) z^{k+p-j}
$$

Let $\alpha_{1}=\sum_{j=1}^{k} s(j) d(j) z^{-j}$ and $\alpha_{2}=\sum_{j=k+1}^{k+p} s(j) d(j) z^{-j}$. Then we have

$$
\left(z^{k+p}-z^{k}\right)\left(1-\alpha_{1}\right)=\alpha_{2} z^{k+p}
$$

and thus

$$
1-\alpha_{1}=\frac{\alpha_{2}}{1-z^{-p}}
$$

Using the geometric series formula yields

$$
1=\alpha_{1}+\sum_{m=0}^{\infty} \alpha_{2} z^{-p m}=\sum_{j=1}^{k} s(j) d(j) z^{-j}+\sum_{m=0}^{\infty} \sum_{j=k+1}^{k+p} s(j) d(j) z^{-p m-j},
$$

and the right hand side is $\sum_{j=1}^{\infty} s(j) d(j) z^{-j}$, showing (3.1). The argument can be reversed to show the opposite implication.

Setting $z=\beta$, the expression for the $(\beta, E)$-expansion of 1 shows that $P_{\beta, E}(\beta)=0$, and it follows that all Galois conjugates of $\beta$ with $|z|>1$ satisfy (3.1).

Remark. We do not know whether $P_{\beta, E}$ is irreducible, so there may be $z$ which satisfy $P_{\beta, E}(z)=0$ but are not Galois conjugates of $\beta$. We call such $z$ the generalized $\beta$-conjugates, following terminology of Verger-Gaugry in the $\beta$-transformation case $[26,25]$. The generalized $\beta$-conjugates also satisfy the equation (3.1). It would be interesting to extend the results of [26, 25] to study the distribution of the generalized $\beta$-conjugates.

Remark. If the $(\beta, E)$-expansion of 1 is finite, the polynomial $P_{\beta, E}$ can be obtained equivalently by looking directly at this finite $(\beta, E)$-expansion; that is, the expression $1=\frac{s(1) d(1)}{\beta}+\frac{s(2) d(2)}{\beta^{2}}+\ldots+\frac{s(n) d(n)}{\beta^{n}}$. In this case, $P_{\beta, E}=$ $z^{n}-\sum_{j=1}^{n} s(j) d(j) z^{n-j}=z^{n}-\sum_{j=1}^{n} s(j) d^{\prime}(j) z^{n-j}-1$.

Remark. Liao and Steiner studied the class of negative $\beta$-transformations in 12. This is the subclass of generalized $\beta$-transformations where the sign of all the branches is set to -1 . They call a number $\beta$ for which the negative 
$\beta$-expansion of 1 is pre-periodic a Yrrap number, and give examples. It is immediate that every Yrrap number is a generalized Parry number.

3.3. Upper bounds on conjugates for PCF $(\beta, E)$-transformations. Combining Lemmas 2.2 and 3.1, we see that any Galois conjugate of a generalized Parry number $\beta$ with $|z|>1$ satisfies

$$
1+\sum_{j=1}^{\infty} c_{j} z^{-j}=\sum_{j=0}^{\infty} c_{j} z^{-j}=0
$$

where $c_{j}=s(j+1) f^{j}(1) \in[-1,1]$ and $f$ is a $\mathrm{PCF}(\beta, E)$-transformation. (The same is true for the generalized $\beta$-conjugates with $|z|>1$ ). Consider the class of functions

$$
\mathcal{F}=\left\{T(w)=1+\sum_{j=1}^{\infty} a_{j} w^{j}: a_{j} \in[-1,1]\right\} .
$$

If $\lambda$ is a zero of a function in $\mathcal{F}$, then $z=\lambda^{-1}$ satisfies

$$
1+\sum_{j=1}^{\infty} a_{j} z^{-j}=0
$$

Thus if $z$ is a Galois conjugate of a generalized Parry number $\beta$ with $|z|>1$, then $z^{-1}$ is a zero of the function in $\mathcal{F}$ with coefficients as in (3.2).

Lemma 3.2. Any zero $\lambda$ of any function in $\mathcal{F}$ has modulus at least $\frac{1}{2}$. If any of the $a_{j}$ satisfy $\left|a_{j}\right|<1$, then $|\lambda|>\frac{1}{2}$.

Proof. Suppose $T(\lambda)=1+\sum_{j=1}^{\infty} a_{j} \lambda^{j}=0$, where $a_{j} \in[-1,1]$. We argue by contradiction. Suppose that $|\lambda|<\frac{1}{2}$. Then $\left|a_{j} \lambda^{j}\right|=\left|a_{j}\right||\lambda|^{j}<2^{-j}$. Thus

$$
\left|\sum_{j=1}^{\infty} a_{j} \lambda^{j}\right| \leq \sum_{j=1}^{\infty}\left|a_{j} \lambda^{j}\right|<\sum_{j=1}^{\infty} 2^{-j}=1,
$$

which contradicts the fact that if $\lambda$ is a zero of $T$, then $\left|\sum_{j=1}^{\infty} a_{j} \lambda^{j}\right|=1$. If we assume further that at least one of the $a_{j}$ satisfy $\left|a_{j}\right|<1$, then for every $\lambda$ with $|\lambda| \leq \frac{1}{2}$ we have $\left|\sum_{j=1}^{\infty} a_{j} \lambda^{j}\right|<1$, so the same contradiction argument applies.

Clearly, this bound is sharp by setting all the $a_{j}=-1$ and letting $\lambda=\frac{1}{2}$.

Theorem 3.3. If $\beta$ is a generalized Parry number, then all Galois conjugates $z$ of $\beta$ satisfy $|z|<2$.

Proof. By Lemmas 2.2 and 3.1, if $z$ is a Galois conjugate of $\beta$ with $|z|>1$, then $z^{-1}$ is a zero of a function in $\mathcal{F}$ whose coefficients are given by $a_{j}=$ $s(j+1) f^{j}(1)$ for the appropriate $(\beta, E)$-transformation $f$. Thus we can apply Lemma 3.2 to show that $|z|<2$. The inequality is strict because the only way we can have $\left|a_{j}\right|=1$ for all $j$ is if 1 is a fixed point of $f$. This can only happen if $\beta$ is an integer, and thus does not have Galois conjugates. 
Remark. For $\beta$-transformations, Solomyak showed [20] that if $z$ is a Galois conjugate of $\beta$ with $|z|>1$, then $z^{-1}$ is a zero of a function in $\mathcal{F}_{[0,1]}$, where

$$
\mathcal{F}_{[0,1]}=\left\{T(w)=1+\sum_{j=1}^{\infty} a_{j} w^{j}: a_{j} \in[0,1]\right\} .
$$

He used this characterization to show that $|z| \leq(\sqrt{5}+1) / 2$ by exploiting the fact that $\left|a_{j}-\frac{1}{2}\right| \leq \frac{1}{2}$.

\section{Conjugates with a prescribed argument and SolomyaK's STRUCTURE THEOREM}

We investigate the maximum possible modulus of a conjugate with a prescribed argument. Let

$\lambda_{\phi}=\min \{|\lambda|: \lambda$ is a zero of a function in $\mathcal{F}$ and the argument of $\lambda$ is $\phi\}$.

A description of the function in $\mathcal{F}$ that attains $\lambda_{\phi}$ is the content of the Solomyak Structure Theorem. Such a function is called $\phi$-optimal. This result was originally established by Solomyak [20] to analyze the zeroes of functions in $\mathcal{F}_{[0,1]}$. The Structure Theorem was generalized by Beaucoup, Borwein, Boyd and Pinner [2] to a class of power series with restricted coefficients that includes $\mathcal{F}$. The following statement is given in [2].

Theorem 4.1 (Solomyak Structure Theorem for $\mathcal{F}$ ). Given an argument $\phi \in(0, \pi)$, there exists $\alpha=\alpha_{\phi} \in(0, \pi)$ and a function

$$
T_{\phi}(w)=1+\sum_{n=1}^{\infty} a_{n} w^{n}
$$

whose coefficients satisfy

$$
a_{n}= \begin{cases}1 & \text { if } n \phi-\alpha \in(0, \pi) \quad(\bmod 2 \pi) \\ -1 & \text { if } n \phi-\alpha \in(-\pi, 0) \quad(\bmod 2 \pi),\end{cases}
$$

such that $T_{\phi}$ is $\phi$-optimal; i.e. $T_{\phi}\left(\lambda_{\phi} e^{i \phi}\right)=0$.

The sequence of coefficients $\left\{a_{n}\right\}$ is determined by the rotation sequence of slope $\phi$ and base point $\alpha$ with the possible exception of one coefficient $a_{j}$, which we call the anomalous coefficient. In the case of $\mathcal{F}_{[0,1]}$, where $\phi$-optimal functions have non-anomalous coefficients belonging to $\{0,1\}$, Solomyak gives explicit examples of $\phi$-optimal functions for which the anomalous coefficient is different from all the rest. The function $T_{\phi}$ is unique when $\phi / 2 \pi$ is irrational, and it is conjectured that when $\phi / 2 \pi$ is rational, $\phi$-optimal functions are never unique, see [20].

No convenient characterizations of the anomalous coefficient or the function $\phi \rightarrow \alpha_{\phi}$ are currently available. Despite these drawbacks, Solomyak put his structure theorem to impressive use in [20, obtaining results on the continuity and differentiability of the function $\phi \rightarrow \lambda_{\phi}$. In particular, we have 
the following result whose proof was given in the $\mathcal{F}_{[0,1]}$ case in [20, Lemma 4.2 ], and was observed to extend almost verbatim to $\mathcal{F}$ in [2, Proposition 1].

Theorem $4.2([20,2])$. The function $\phi \rightarrow \lambda_{\phi}$ is continuous on $(0, \pi)$.

We apply this result in the following theorem.

Theorem 4.3. The quantity $\lambda_{\phi}$ is bounded uniformly away from $\frac{1}{2}$ for $\phi \in$ $(0, \pi)$. Thus, $\sup \left\{\lambda_{\phi}^{-1}: \phi \in(0, \pi)\right\}<2$.

Proof. First, we show that $\lambda_{\phi}>\frac{1}{2}$ on $(0, \pi)$. Suppose not. Then there exists $\theta \in(0, \pi)$, and $\lambda=\frac{1}{2} e^{i \theta}$, and $a_{n} \in[-1,1]$ so that

$$
1+\sum a_{n} \lambda^{n}=0
$$

Furthermore, by Lemma $\left[3.2, a_{n} \in\{1,-1\}\right.$ for all $n$. In particular, writing

$$
a_{n} \lambda^{n}=\frac{1}{2^{n}} e^{i \theta_{n}}
$$

we have

$$
1+\sum_{n=1}^{\infty} \frac{1}{2^{n}} e^{i \theta_{n}}=0
$$

Thus

$$
1+\frac{1}{2} e^{i \theta_{1}}=-\sum_{n=2}^{\infty} \frac{1}{2^{n}} e^{i \theta_{n}} .
$$

Letting $z_{1}=1+\frac{1}{2} e^{i \theta_{1}}$. we see that $\left|z_{1}-1\right|=\frac{1}{2}$. Let $z_{2}=-\sum_{n=2}^{\infty} \frac{1}{2^{n}} e^{i \theta_{n}}$. Then $\left|z_{2}\right| \leq \sum_{n=2}^{\infty} \frac{1}{2^{n}}=\frac{1}{2}$. Thus, if $z_{1}=z_{2}$, then $z_{1}=\frac{1}{2}$, and so $\theta_{1}=\pi$. Thus we have $a_{1} \lambda=-\frac{1}{2}$. Since $a_{1} \in\{1,-1\}$, it follows that $\lambda=\frac{1}{2}$ or $\lambda=-\frac{1}{2}$. This contradicts the hypothesis that $\lambda=\frac{1}{2} e^{i \theta}$ for $\theta \in(0, \pi)$.

Since the map $\phi \rightarrow \lambda_{\phi}$ is continuous on $(0, \pi)$, all that remains is to analyze the limit of $\lambda_{\phi}$ as $\phi \rightarrow 0$ and $\phi \rightarrow \pi$. The argument of [20, Lemma 4.1] shows that $\lim _{\phi \rightarrow 0} \lambda_{\phi}$ converges to a value which is a double root of some function in $\mathcal{F}$, and likewise for $\lim _{\phi \rightarrow \pi} \lambda_{\phi}$. Solomyak gives a rigorous argument that the smallest double root of a function in $\mathcal{F}$ is greater than 0.6299 on p.622 of [21]. Thus $\min \left\{\lim _{\phi \rightarrow \pi} \lambda_{\phi}, \lim _{\phi \rightarrow 0} \lambda_{\phi}\right\} \geq 0.6299>\frac{1}{2}$. It follows that $\sup \left\{\lambda_{\phi}^{-1}: \phi \in(0, \pi)\right\}<2$.

Remark. Note that $\lambda_{0}=\lambda_{\pi}=\frac{1}{2}$. This is because $\lambda=\frac{1}{2}$ is a zero of the function in $\mathcal{F}$ with $a_{n}=-1$ for all $n$, and $\lambda=-\frac{1}{2}$ is a zero of the function in $\mathcal{F}$ with $a_{n}=(-1)^{n+1}$ for all $n$. Thus the function $\phi \rightarrow \lambda_{\phi}$ is discontinuous at 0 and $\pi$.

Remark. The function $\phi \rightarrow \lambda_{\phi}$ is investigated numerically in [2], and is plotted as figure 2 of [2]. The numerics suggest that $\lambda_{\phi} \in(0.63,0.71)$ for $\phi \in(0, \pi)$, and that $\lim _{\phi \rightarrow \pi} \lambda_{\phi} \approx 0.6491$. Thus, we should expect that $\sup \left\{\lambda_{\phi}^{-1} \mid \phi \in(0, \pi)\right\}<1.59$. It is an open question to rigorously establish optimal bounds on this quantity. 
Remark. Solomyak studied differentiability properties of the boundary of $\mathcal{F}_{[0,1]}$ in some detail using the tools introduced above, giving criterion for when $\phi \rightarrow \lambda_{\phi}$ is smooth, and non-smooth. The extension of these results to $\mathcal{F}$ is claimed in [2, Proposition 1], with proof referred to Solomyak.

\section{THE SET $\bar{\Omega}$}

Recall that $\Omega$ is defined to be the set of all Galois conjugates for all generalized Parry numbers. Let $\mathcal{G}$ denote the set of zeroes of functions in $\mathcal{F}$. We have shown that $\{z \in \Omega|| z \mid>1\} \subset\left\{z \mid z^{-1} \in \mathcal{G}\right\}$. In this section, our main result is a partial converse of this: we will show that $\left\{z \mid z^{-1} \in \mathcal{G}\right\} \subset\{z \in \bar{\Omega}|| z \mid>1\}$. To prove this, first we require a means of identifying when a number is a generalized Parry number.

5.1. Criteria for $\beta$ to be a generalized Parry number. Suppose that $((s(1), a(1)), \ldots,(s(n), a(n)))$ is a finite sequence where $n \geq 2$, the $a(j)$ are non-negative integers with $a(n) \neq 0$, the $s(j) \in\{1,-1\}$, and $s(1)=1$. Let the finite sequence $(\operatorname{It}(1), \ldots, \operatorname{It}(n))$ be given by

$$
\operatorname{It}(j)= \begin{cases}a(j) & \text { if } s(j+1)=s(j) \\ a(j)-1 & \text { if } s(j+1)=-s(j) .\end{cases}
$$

for $j \in\{1, \ldots, n-1\}$, and $\operatorname{It}(n)=a(n)$. We impose the following hypotheses on the sequence:

(1) $\operatorname{It}(1)>a(j)$ for all $j \geq 2$;

(2) there exists a sign configuration $E \in\{1,-1\}^{m+1}$, where $m=\operatorname{It}(1)$, such that $s(j+1)=s(j) E(\operatorname{It}(j))$ for each $j \in\{1, \ldots, n-1\}$.

(3) if $a(j)=0$, then $s(j+1)=s(j)$, to ensure that $\operatorname{It}(j) \geq 0$. Thus, if any of the $a(j)$ are 0 , then $E(0)=1$.

Writing $w=(\operatorname{It}(1), \ldots, \operatorname{It}(n))$, we will find a $\beta$ such that the itinerary of 1 for $f_{\beta, E}$ is either $w^{\infty}$ or $w 0^{\infty}$.

A complete characterization of which sequences arise as the itinerary of 1 for some $(\beta, E)$-transformation is currently open. A statement for $\operatorname{It}(1) \geq 2$ appears as Theorem 25 of Góra [10], although his hypotheses have been criticized by Steiner in [22]. We do not pursue the general case here since we are investigating only PCF transformations.

We define the function

$$
F(x)=s(1) a(1)+\frac{s(2) a(2)}{x}+\frac{s(3) a(3)}{x^{2}}+\ldots+\frac{s(n) a(n)}{x^{n-1}} .
$$

We want to show that $F$ has a fixed point in the interval $(\operatorname{It}(1), \operatorname{It}(1)+1)$. To this end, for $j \in\{1, \ldots, n-1\}$, let

$$
R_{j}(x)=\sum_{i=j}^{n-1} \frac{s(i+1) a(i+1)}{x^{i}} .
$$

Lemma 5.1. For $x \in[\operatorname{It}(1), \operatorname{It}(1)+1]$, and $j \in\{1, \ldots, n-1\}$, we have 
(1) $\left|R_{j}(x)\right|<\frac{1}{x^{j-1}}$

(2) $\operatorname{sign}\left(R_{j}(x)\right)=s(j+1)$.

Proof. Let $N=\max \{a(2), \ldots, a(n)\}$. Then, since $N \leq \operatorname{It}(1)-1 \leq x-1$,

$$
\left|R_{j}(x)\right|<\sum_{i=j}^{\infty} \frac{N}{x^{i}}=\frac{1}{x^{j-1}} \frac{N}{x-1} \leq \frac{1}{x^{j-1}} .
$$

Now take the first $k \geq 1$ so that $a(j+k) \neq 0$. If $k \geq 2$, then since $a(j+1)=\cdots=a(j+k-1)=0$, we have $s(j+k)=s(j+1)$. We have

$$
R_{j}(x)=\frac{s(j+k) a(j+k)}{x^{j+k-1}}+R_{j+k}(x),
$$

and thus

$$
\frac{s(j+k) a(j+k)}{x^{j+k-1}}-\frac{1}{x^{j+k-1}}<R_{j}(x)<\frac{s(j+k) a(j+k)}{x^{j+k-1}}+\frac{1}{x^{j+k-1}} .
$$

Since $a(j+k) \geq 1$, it follows that $\operatorname{sign}\left(R_{j}(x)\right)=s(j+k)=s(j+1)$.

It follows immediately from (2) in Lemma 5.1 that

$$
\left|R_{j}(x)\right|=s(j+1) R_{j}(x) .
$$

Lemma 5.2. There exists $\beta \in(\operatorname{It}(1), \operatorname{It}(1)+1)$ such that

$$
\beta=s(1) a(1)+\frac{s(2) a(2)}{\beta}+\frac{s(3) a(3)}{\beta^{2}}+\ldots+\frac{s(n) a(n)}{\beta^{n-1}}
$$

Proof. We show that $F:[\operatorname{It}(1), \operatorname{It}(1)+1] \rightarrow[\operatorname{It}(1), \operatorname{It}(1)+1]$. Note that $F(x)=a(1)+R_{1}(x)$. There are two cases. First suppose $s(2)=1$. Then $E(\operatorname{It}(1))=1$ and $\operatorname{It}(1)=a(1)$. Since $0<R_{1}(x)<1$, we have

$$
\operatorname{It}(1)+1=a(1)+1>a(1)+R_{1}(x)>a(1)=\operatorname{It}(1) .
$$

Now suppose $s(2)=-1$. Then $E(\operatorname{It}(1))=-1$ and $\operatorname{It}(1)=a(1)-1$. Since $0>R_{1}(x)>-1$, we have

$$
\operatorname{It}(1)+1=a(1)>a(1)+R_{1}(x)>a(1)-1=\operatorname{It}(1) .
$$

Thus, in both cases, the image of $F$ is contained in $(\operatorname{It}(1), \operatorname{It}(1)+1)$. Considering the map as $F:[\operatorname{It}(1), \operatorname{It}(1)+1] \rightarrow[\operatorname{It}(1), \operatorname{It}(1)+1]$, it follows from the Intermediate Value Theorem that $F$ has a fixed point $\beta$. Clearly, $\beta \notin\{\operatorname{It}(1), \operatorname{It}(1)+1\}$.

From now on, we fix $\beta \in(\operatorname{It}(1), \operatorname{It}(1)+1)$ provided by Lemma 5.2, and let $f=f_{\beta, E}$. Recall that $[0,1]$ is partitioned into intervals $I_{0}=[0,1 / \beta]$, $I_{1}=(1 / \beta, 2 / \beta], \ldots, I_{m}=(m / \beta, 1]$, where $m=\operatorname{It}(1)$.

Lemma 5.3. For $k \in\{1, \ldots, n-1\}$, we have

(i) $f^{k-1}(1) \in I_{\mathrm{It}(k)}$

(ii) $f^{k-1}(1)=\frac{1}{\beta}\left(a(k)+\beta^{k-1} E(\operatorname{It}(k))\left|R_{k}(\beta)\right|\right)$ 
Proof. We argue recursively. For $k=1$, it is immediate that $1 \in I_{\mathrm{It}(1)}$, and we have

$$
\begin{aligned}
1 & =\frac{s(1) a(1)}{\beta}+\frac{s(2) a(2)}{\beta^{2}}+\ldots+\frac{s(n) a(n)}{\beta^{n}} \\
& =\frac{a(1)}{\beta}+\frac{1}{\beta} R_{1}(\beta),
\end{aligned}
$$

and since $R_{1}(\beta)=s(2)\left|R_{1}(\beta)\right|=E(\operatorname{It}(1))\left|R_{1}(\beta)\right|$, we are done.

Now we show that if $k \in\{1, \ldots, n-1\}$, and $(i)$ and $(i i)$ hold true for $f^{k-1}(1)$, then

$$
f^{k}(1)=\beta^{k-1}\left|R_{k}(\beta)\right|
$$

There are two cases:

Case (a): Suppose that $s(k+1)=s(k)$. Then $a(k)=\operatorname{It}(k)$, and $E(\operatorname{It}(k))=1$. Since $E(\operatorname{It}(k))=1$, for $y \in I_{\operatorname{It}(k)}, f(y)=\beta y-a(k)$, and thus applying $f$ to $f^{k-1}(1) \in I_{\mathrm{It}(k)}$, it follows from the expression $(i i)$ that $f^{k}(1)=\beta^{k-1}\left|R_{k}(\beta)\right|$.

Case (b): Suppose that $s(k+1)=-s(k)$. Then $a(k)-1=\operatorname{It}(k)$, and $E(\operatorname{It}(k))=-1$. Since $E(\operatorname{It}(k))=-1$, for $y \in I_{\operatorname{It}(k)}, f(y)=a(k)-\beta y$. Since $f^{k-1}(1) \in I_{\mathrm{It}(k)}$, it follows from the expression $(i i)$ that $f^{k}(1)=$ $-E(\operatorname{It}(k)) \beta^{k-1}\left|R_{k}(\beta)\right|=\beta^{k-1}\left|R_{k}(\beta)\right|$.

Now fix $k \in\{1, \ldots, n-2\}$, and suppose that $(i)$ and $(i i)$ hold true for $f^{k-1}(1)$. It follows from (5.1) and (5.2) that

$$
\begin{aligned}
f^{k}(1) & =\beta^{k-1}\left|R_{k}(\beta)\right|=\beta^{k-1} s(k+1) R_{k}(\beta) \\
& =\beta^{k-1} s(k+1)\left(\frac{s(k+1) a(k+1)}{\beta^{k}}+R_{k+1}(\beta)\right) \\
& =\frac{1}{\beta}\left(a(k+1)+\beta^{k} s(k+1) R_{k+1}(\beta)\right) \\
& =\frac{1}{\beta}\left(a(k+1)+\beta^{k} s(k+1) s(k+2)\left|R_{k+1}(\beta)\right|\right) .
\end{aligned}
$$

Since $s(k+1) s(k+2)=s(k+1)^{2} E(\operatorname{It}(k+1))$, we have established the formula (ii) for $f^{k}(1)$.

Now, we show that $(i i)$ implies $(i)$. Again, there are two cases. If $s(k+$ $2)=s(k+1)$, then $a(k+1)=\operatorname{It}(k+1)$, and $E(\operatorname{It}(k+1))=1$, so

$$
\frac{a(k+1)}{\beta}<f^{k}(1)=\frac{1}{\beta}\left(a(k+1)+\beta^{k}\left|R_{k+1}(\beta)\right|\right)<\frac{a(k+1)+1}{\beta},
$$

and so $f^{k}(1) \in I_{\mathrm{It}(k+1)}$.

In the case that $s(k+2)=-s(k+1)$, then $a(k+1)-1=\operatorname{It}(k+1)$, and $E(\operatorname{It}(k+1))=-1$, so

$$
\frac{a(k+1)-1}{\beta}<f^{k}(1)=\frac{1}{\beta}\left(a(k+1)-\beta^{k}\left|R_{k+1}(\beta)\right|\right)<\frac{a(k+1)}{\beta},
$$


and so $f^{k}(1) \in I_{\mathrm{It}(k+1)}$.

This shows that both $(i)$ and $(i i)$ are true for $f^{k}(1)$, which completes the proof.

Lemma 5.4. We have $f^{n-1}(1)=a(n) / \beta$. Thus $f$ is $P C F$, and so $\beta$ is a generalized Parry number.

Proof. Since by Lemma 5.3, $(i)$ and (ii) hold for $f^{n-2}(1)$, the equation (5.2) shows that $f^{n-1}(1)=\beta^{n-2}\left|R_{n-1}(\beta)\right|=a(n) / \beta$. Since $f(a(n) / \beta)$ is either 1 or 0 (noting that $a(n) / \beta \in I_{a(n)-1}$, so this depends only on whether $E(a(n)-1)$ is positive or negative), we have shown that 1 has a finite orbit, and hence $f$ is PCF.

The previous results allow us to give the following simple criterion for finding generalized Parry numbers.

Theorem 5.5. Suppose that $M(1), \ldots M(n)$ are distinct non-zero integers such that $M(1) \geq 2,|M(j)|+1<M(1)$ for all $j \geq 2$, and $|M(j)| \neq$ $|M(k)|-1$ for all $j, k$. Then the equation

$$
x=M(1)+\frac{M(2)}{x}+\frac{M(3)}{x^{2}}+\ldots+\frac{M(n)}{x^{n-1}}
$$

has a solution $\beta>1$ which is a generalized Parry number.

Proof. Let $a(j)=|M(j)| \geq 1$ and $s(j)=\operatorname{sign}(M(j))$, and $\operatorname{define~} \operatorname{It}(j)$ as we did earlier in this section. Our hypotheses imply that $\operatorname{It}(1)>a(j)$ for all $j \geq 2$. Since all the $\operatorname{It}(j)$ are distinct, we have freedom to choose a vector $E$ whose entries in the positions $E(\operatorname{It}(j))$ leads to the sequence of signs $s(j)$. Thus applying Lemma 5.2, (5.3) has a solution $\beta \in(\operatorname{It}(1), \operatorname{It}(1)+1)$, and so by Lemma 5.4, $f_{\beta, E}$ is PCF, and thus $\beta$ is a generalized Parry number.

Remark. We mention some other classes of numbers that are known to be Parry numbers, and hence generalized Parry numbers. Schmidt proved that if $\beta$ is a Pisot number, then $\beta$ is Parry [19]. Whether Salem numbers are Parry is a challenging open problem [4, 23], first raised by Schmidt in [19]. Boyd proved that degree 4 Salem numbers are Parry [3]. Numerical evidence and hueristic arguments by Thurston [23] and Boyd suggest that most higher degree Salem numbers are not Parry, but it seems to be very difficult to find even a single rigorous example of this phenomenon.

5.2. Characterization of $\bar{\Omega}$. We now prove our main result about $\bar{\Omega}$.

Theorem 5.6. The set $\bar{\Omega}$ is $\mathbb{D} \cup\left\{z|| z \mid>1\right.$ and $\left.z^{-1} \in \mathcal{G}\right\}$, where $\mathbb{D}=\{z \mid$ $|z| \leq 1\}$.

Proof. We already know that the closed unit disk $\mathbb{D}$ is a subset of $\bar{\Omega}$, because $\mathbb{D}$ is contained in the closure of the Galois conjugates of the simple Parry numbers (see Theorem 2.1 of [20]). Thus, all it remains to show is that if $|z|>1$ and $z^{-1} \in \mathcal{G}$, then $z \in \bar{\Omega}$. The argument is a generalization of the second half of the proof of [20, Theorem 2.1]. 
Let $\lambda=z^{-1}$. Then $T(\lambda)=0$ for some $T(w)=1+\sum_{j=1}^{\infty} a_{j} w^{j}$ with $a_{j} \in[-1,1]$. We approximate $T$ with a function $g(w)=1+\sum_{j=1}^{n-1} b_{j} w^{j}$ with $b_{j} \in(-1,1)$, where all $b_{j}$ are rational. By taking $n$ large, and the $b_{j}$ arbitrarily close to the $a_{j}$, we can ensure that $g$ has a zero arbitrarily close to $\lambda$. Writing each $b_{j}$ in the form $b_{j}=M(j+1) / M(1)$, where $M(n) \in \mathbb{Z}$, we have

$$
g(w)=M(1)^{-1}\left(M(1)+M(2) w+\ldots+M(n) w^{n-1}\right) .
$$

We can make sure that our $b_{j}$ are chosen so that all $M(j)$ are distinct and non-zero, $M(1) \geq 2,|M(j)|+1<M(1)$ for all $j$, and $|M(j)| \neq|M(k)|-1$ for all $j, k$. Now take a large prime $p>M(n)$ (to be fixed later). We know by Theorem 5.5 that the equation

$$
x=p M(1)+\frac{p M(2)}{x}+\frac{p M(3)}{x^{2}}+\ldots+\frac{p M(n)}{x^{n-1}}
$$

has a solution $\beta>1$ which is a generalized Parry number. Define the polynomial

$$
q(w)=w^{n}-p M(1) w^{n-1}-p M(2) w^{n-2}-\ldots-p M(n),
$$

so that $\beta$ is a zero of $q$. The polynomial $q$ is irreducible by Eisenstein's Criterion, and hence $q$ is the minimal polynomial for $\beta$. Thus all other zeroes of $q$ are conjugates of $\beta$.

The rest of the argument is to show that one of these zeroes is close to z. Elementary computation shows that $q\left(w^{-1}\right)=0$ if and only if $g(w)=$ $\frac{1}{w p M(1)}$. Thus letting $h(w)=-\frac{1}{p M(1) w}$, we have $q\left(w^{-1}\right)=0$ if and only if $(g+h)(w)=0$. We now use Rouché's theorem. We are free to choose $p$ as large as we like, so we can ensure that $|h|<|g|$ on a small circle $\gamma$ centered at $\lambda$. Thus, $g+h$ has the same number of zeroes as $g$ inside $\gamma$. By the choice of $g$, we can ensure that $g$, and hence $g+h$, has a zero $w_{0}$ inside $\gamma$. Thus, $q$ has a zero $w_{0}^{-1}$ with $w_{0}$ in a neighbourhood of $\lambda$. We can ensure that $w_{0}$, which by construction is the inverse of a Galois conjugate of a simple generalized Parry number, is arbitrarily close to $\lambda$. This completes the proof.

5.3. Path-connectedness of $\bar{\Omega}$. Given Theorem 5.6, it is now easy to show that $\bar{\Omega}$ is path connected. We use the following lemma.

Lemma 5.7. Let $z=r e^{i \theta} \in \bar{\Omega}$ with $r>1$. Then for all $r^{\prime} \in(1, r)$, $z^{\prime}=r^{\prime} e^{i \theta} \in \bar{\Omega}$.

Proof. By Theorem [5.6, we have $\lambda=z^{-1} \in \mathcal{G}$, so $T(\lambda)=0$ for some $T \in \mathcal{F}$. For any $a>1$, the function $\bar{T}(z):=T(z / a) \in \mathcal{F}$, and has $a \lambda$ as a zero. Thus $a \lambda \in \mathcal{G}$, and so $\frac{1}{a} z \in \bar{\Omega}$ for any $a>1$.

Thus, we can connect any two points in $\bar{\Omega}$ using, for example, paths along at most two radial lines together with a path along the unit circle $S^{1}$. 


\section{UNIMODAL MAPS AS GENERALIZED $\beta$-TRANSFORMATIONS}

We now use the results of the previous sections to study topological entropy for PCF continuous unimodal maps. The topological entropy of a continuous map on a compact metric space is a number that captures the exponential growth rate of distinct orbits of length $n$, and is a fundamental invariant of a topological dynamical system. See Walters for a general definition 27. The problem of deciding if a number can be obtained as the entropy of a map from a given class of systems has a long history with notable results including [13, 11, 23].

For piecewise monotonic interval maps, the topological entropy has a simple formula which was first obtained by Misiurewecz and Szlenk [15, 1], and which for current purposes we take as our definition.

Definition 6.1. Let $f$ be a piecewise monotonic map of the unit interval. The topological entropy, which we denote $h_{\mathrm{top}}(f)$, or simply $h$, is defined to be

$$
h_{\mathrm{top}}(f)=\lim _{n \rightarrow \infty} \frac{1}{n} \log \#\left\{\text { branches of monotonicity for } f^{n}\right\} .
$$

Note that it is immediate from the definition that the entropy of a unimodal map is at most $\log 2$. One can prove that if $f$ is a generalized $\beta$ transformation, then $h_{\text {top }}(f)=\log \beta$, see e.g. Corollary 4.3.13 of [1]. It is well known that every unimodal map $f$ is topologically semi-conjugate to a $\lambda$-uniform expander $g$; that is, a piecewise affine continuous interval map whose slope on each interval of monotonicity is either $\lambda$ or $-\lambda$. In particular, $h_{\mathrm{top}}(f)=h_{\mathrm{top}}(g)$, and if $f$ is PCF, then $g$ is PCF. This result was first proved in [14], and is given as Theorem 4.6.8 of [1]. Thus, to study the entropies of PCF unimodal maps, it suffices to study the entropies of PCF uniform expanders. Unimodal uniform expanders (perhaps after modifying by a conjugacy) can be thought of as generalized $\beta$-transformations. Thus the formalism of generalized $\beta$-transformations can be used to study the entropy of unimodal maps. In particular, we have the following lemma.

Lemma 6.1. Every PCF unimodal map is conjugate to a PCF generalized $\beta$-transformation

Proof. It suffices to show that every PCF $\lambda$-uniform expander $g$ is conjugate to a PCF generalized $\beta$-transformation. First, we use the standard trick of trimming the domain of $g$, and rescaling to get a surjective map of the unit interval. Let $\Lambda=\bigcap g^{i}([0,1])=[a, b]$, and consider $\left.g\right|_{\Lambda}$. The critical point $c$ satisfies either $g(c)=a$ or $g(c)=b$. We also have $a, b \in\{g(a), g(b), g(c)\}$. We can conjugate by the affine transformation $\pi(x)=\frac{1}{b-a}(x-a)$ to the map $G(x)=\pi \circ g \circ \pi^{-1}(x)=\frac{1}{b-a}[g((b-a) x+a)-a]$. Clearly an affine transformation will send critical points to critical points, and a conjugacy sends periodic orbits to periodic orbits, so the new map $G$ is PCF, surjective, and has domain $[0,1]$. 
To be surjective, $G$ must have at least one full branch. There are four possibilities:

(1) first branch full; sign configuration $(1,-1)$;

(2) first branch full; sign configuration $(-1,1)$;

(3) second branch full; sign configuration $(-1,1)$;

(4) second branch full; sign configuration $(1,-1)$.

Cases (1) and (2) are generalized $\beta$-transformations, with $\beta=\lambda$, and the appropriate sign configuration. For case (3), we conjugate by the transfomation $\pi(x)=-x$ to get the transfomation $h(x)=-G(-x)$ defined on $[-1,0]$. We can conjugate by a translation to return the domain to $[0,1]$. The new map is in case (2). Similarly, a map in case (4) is conjugate to a map in case (1). Thus, $G$ is either a generalized $\beta$-transformation, or conjugate to a generalized $\beta$-transformation by an affine transformation.

Thus, for a PCF unimodal map $f$, we have $h_{\mathrm{top}}(f)=\log \beta$ for some generalized Parry number $\beta \in[1,2]$. So we have

$$
\Omega_{T}:=\left\{z \mid z \text { is a conjugate of } e^{h(f)} \text { for a PCF unimodal map } f\right\} \subset \Omega .
$$

The problem of giving a description of $\Omega_{T}$ was raised in Thurston's final paper [23. His numerical results showed that apart from a spike along the real axis, this set appears to lie in a disk much smaller than the disk $|z|<2$. Our description of $\Omega$ allows us to conclude that $\Omega_{T} \backslash \mathbb{R}$ indeed lies in a disk of radius strictly less than 2. As mentioned previously, although numerical results suggest that we should expect a bound less than 1.59, rigorous sharp bounds are currently out of reach. Nevertheless, we establish the principle that all non-real Galois conjugates are contained inside a disk with a smaller radius than the trivial bound 2 . We now state this as a theorem. The proof is an immediate consequence of the discussion above and Theorem 4.3 ,

Theorem 6.2. There exists $\epsilon>0$ so that if $z$ is a conjugate of $e^{h(f)}$ for a $P C F$ unimodal map $f$ and $z \notin \mathbb{R}$, then $|z|<2-\epsilon$.

Many questions remain about the sets $\Omega$ and $\Omega_{T}$. For example, can one describe $\bar{\Omega} \backslash \bar{\Omega}_{T}$ ? One way in which these sets differ is that, from Thurston's picture, $\bar{\Omega}_{T}$ appears to have 'holes' around some roots of unity. This is ruled

out for $\bar{\Omega}$ by the star-convexity proved in Lemma 5.7. It would be interesting to determine the exact location and distribution of the holes that appear in $\bar{\Omega}_{T}$. The existence of holes for $\bar{\Omega}_{T}$ inside the unit disk was established in [5]. Another question is to determine where, and by how much, the outer boundaries of $\bar{\Omega}$ and $\bar{\Omega}_{T}$ differ. Numerical investigation of these questions could be a good first step towards rigorous results.

\section{REFERENCES}

[1] Lluís Alsedà, Jaume Llibre, and Michał Misiurewicz. Combinatorial dynamics and entropy in dimension one, volume 5 of Advanced Series in Nonlinear Dynamics. World Scientific Publishing Co., Inc., River Edge, NJ, second edition, 2000. 
[2] Franck Beaucoup, Peter Borwein, David W. Boyd, and Christopher Pinner. Power series with restricted coefficients and a root on a given ray. Math. Comp., 67(222):715736, 1998.

[3] David W. Boyd. Salem numbers of degree four have periodic expansions. In Théorie des nombres (Quebec, $P Q, 1987$ ), pages 57-64. de Gruyter, Berlin, 1989.

[4] M. Boyle. Open problems in symbolic dynamics. Contemp. Math., 469:69-118, 2008.

[5] D. Calegari, S. Koch, and D. Walker. Roots, schottky semigroups, and a proof of bandts conjecture. To appear in Ergodic Theory and Dynamical Systems, 2016.

[6] K. Dajani and C. Kraaikamp. Ergodic Theory of Numbers, volume 29 of Carus mathematical monographs. Mathematical Association of America, Washington D.C., 2002.

[7] Karma Dajani, Yusuf Hartono, and Cor Kraaikamp. Mixing properties of $(\alpha, \beta)$ expansions. Ergodic Theory Dynam. Systems, 29(4), 2009.

[8] B. Faller and C.-E. Pfister. A point is normal for almost all maps $\beta x+\alpha \bmod 1$ or generalized $\beta$-transformations. Ergodic Theory Dynam. Systems, 29(5):1529-1547, 2009.

[9] Natalie Priebe Frank and E. Arthur Robinson, Jr. Generalized $\beta$-expansions, substitution tilings, and local finiteness. Trans. Amer. Math. Soc., 360(3):1163-1177 (electronic), 2008.

[10] Paweł Góra. Invariant densities for generalized $\beta$-maps. Ergodic Theory Dynam. Systems, 27(5):1583-1598, 2007.

[11] Michael Hochman and Tom Meyerovitch. A characterization of the entropies of multidimensional shifts of finite type. Ann. of Math. (2), 171(3):2011-2038, 2010.

[12] Lingmin Liao and Wolfgang Steiner. Dynamical properties of the negative betatransformation. Ergodic Theory Dynam. Systems, 32(5):1673-1690, 2012.

[13] D. A. Lind. The entropies of topological Markov shifts and a related class of algebraic integers. Ergodic Theory Dynam. Systems, 4(2):283-300, 1984.

[14] John Milnor and William Thurston. On iterated maps of the interval. In Dynamical systems (College Park, MD, 1986-87), volume 1342 of Lecture Notes in Math., pages 465-563. Springer, Berlin, 1988.

[15] M. Misiurewicz and W. Szlenk. Entropy of piecewise monotone mappings. Studia Math., 67(1):45-63, 1980.

[16] W. Parry. On the $\beta$-expansions of real numbers. Acta Math. Hung., 11:401-416, 1960.

[17] W. Parry. Representations for real numbers. Acta Math. Acad. Sci. Hungar., 15:95105, 1964.

[18] E. A. Robinson. Parry's topological transitivity and $f$-expansions. To appear in Proc. Amer. Math. Soc., 2015.

[19] Klaus Schmidt. On periodic expansions of Pisot numbers and Salem numbers. Bull. London Math. Soc., 12(4):269-278, 1980.

[20] Boris Solomyak. Conjugates of beta-numbers and the zero-free domain for a class of analytic functions. Proc. London Math. Soc. (3), 68(3):477-498, 1994.

[21] Boris Solomyak. On the random series $\sum \pm \lambda^{n}$ (an Erdős problem). Ann. of Math. (2), 142(3):611-625, 1995.

[22] W. Steiner. Digital expansions with negative real bases. Acta Math. Hungar., 139(12):106-119, 2013.

[23] William P. Thurston. Entropy in dimension one. In Frontiers in complex dynamics, volume 51 of Princeton Math. Ser., pages 339-384. Princeton Univ. Press, Princeton, NJ, 2014

[24] Giulio Tiozzo. Galois conjugates of entropies of real unimodal maps. arXiv preprint arXiv:1310.7647, 2013.

[25] Jean-Louis Verger-Gaugry. On the dichotomy of Perron numbers and beta-conjugates. Monatsh. Math., 155(3-4):277-299, 2008. 
[26] Jean-Louis Verger-Gaugry. Uniform distribution of Galois conjugates and betaconjugates of a Parry number near the unit circle and dichotomy of Perron numbers. Unif. Distrib. Theory, 3(2):157-190, 2008.

[27] Peter Walters. An introduction to ergodic theory, volume 79 of Graduate Texts in Mathematics. Springer-Verlag, New York, 1982.

Department of Mathematics, The Ohio State University, 100 Math Tower, 231 West 18th Avenue, Columbus, Ohio 43210

E-mail address: thompson@math.osu.edu 